Original Article

\title{
Effects of a facilitating device on pelvic floor muscle contraction during breathing exercises
}

\author{
Atsushi Kubota, PhD ${ }^{1 *}$, Keishoku Sakuraba, PhD, MD²), Kazutaka Araki, MS ${ }^{3)}$, \\ Toshimitsu Ishizuka, MS, ATC ${ }^{4)}$, Atsuko Nakanilda, PhD, ATC ${ }^{1)}$, Yoshio Suzuki, PhD ${ }^{5}$ ) \\ 1) Sports Medicine, School of Health and Sports Science, Graduate School of Health and Sports \\ Science, Juntendo University: 1-1 Hiragagakuendai, Inzai, Chiba 270-1695, Japan \\ 2) Department of Sports Medicine, Graduate School of Health and Sports Science, Juntendo \\ University, Japan \\ 3) School of Health Professions, Texas Tech University Health Sciences Center, USA \\ 4) Japan Core Conditioning Association, Japan \\ 5) Sports Nutrition and Biochemistry, Graduate School of Health and Sports Science, Juntendo \\ University School of Health and Sports Science, Japan
}

\begin{abstract}
Purpose] This study aimed to clarify the effects and to verify the efficacy of various breathing exercises performed while sitting on a small foam roller on the contraction of pelvic floor muscles in males. [Participants and Methods] This study, involving 10 healthy males (age $19.9 \pm 1.6$ years), had a crossover design and involved two conditions: sitting at rest for $10 \mathrm{~min}$ (CON condition) and sitting on a small foam roller placed on a chair while performing seven breathing exercises (EXE condition). Movement of the posterior side of the bladder was examined in both conditions using ultrasonic imaging. Pelvic floor muscle contraction was evaluated based on the movement distance. [Results] No significant difference was found in any parameter for CON conditions. The distance of bladder posterior side movement was $5.58 \pm 2.51 \mathrm{~mm}$ (pre), $13.66 \pm 5.16 \mathrm{~mm}$ (post), and $9.59 \pm 3.67 \mathrm{~mm}$ (post-1 month) for EXE conditions. Subjective evaluation also demonstrated that the feeling of contraction was stronger immediately after the experiment. [Conclusion] Results demonstrated that various breathing exercises, performed while sitting on a small foam roller, enhanced the voluntary contraction of pelvic floor muscles in males. Efficacy was demonstrated, at least in young males.

Key words: Chair exercise, Pelvic floor muscles, Voluntary contraction
\end{abstract}

(This article was submitted Jun. 13, 2018, and was accepted Sep. 17, 2018)

\section{INTRODUCTION}

The muscles of the trunk are classified into the outer unit, consisting of outer muscles, and the inner unit, consisting of inner muscles attached to the lumbar vertebrae including the diaphragm, the transverse abdominal muscle, the erector spinae muscle (especially multifidus muscle), and pelvic floor muscles ${ }^{1)}$. The inner unit functions to increase abdominal pressure through cooperation of the muscles, and contributes to spine stabilization and load reduction. Moreover, it has been pointed out that trunk muscles will be unbalanced only when outer muscles of the trunk are trained without training inner muscles, resulting in the risk of injury ${ }^{2}$. For that reason, training the inner unit is also important to prevent injury through various exertions for sports ${ }^{3-6}$. Furthermore, training the inner unit is also attracting attention for lower back pain reduction as well as maintenance and improvement of body type for people without exercise habits ${ }^{7}$.

Pelvic floor muscles, part of the inner unit of muscles, have been attracting attention particularly because they are presum-

*Corresponding author. Atsushi Kubota (E-mail: akubota@juntendo.ac.jp)

(C2018 The Society of Physical Therapy Science. Published by IPEC Inc.

(c) (1) $\odot$ This is an open-access article distributed under the terms of the Creative Commons Attribution Non-Commercial No Deriva-

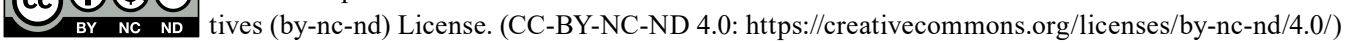


ably involved in incontinence in females. Earlier reports show that pelvic floor muscles are involved in increased abdominal pressure ${ }^{8)}$. Therefore, pelvic floor muscle contraction is important for both males and females. However, the movement of pelvic floor muscles has not been fully verified. Moreover, no exercise that can be performed by everyone can be expected to show beneficial effects for them. The improvement of exercise equipment is progressing. A tool that might be effective to promote pelvic floor muscle contraction was developed: a small, 20.5-cm-long, 4-cm-diameter foam roller. An ordinary foam roller (Stretch Pole ${ }^{\circledR} ; \mathrm{LPN}$ ) is $98 \mathrm{~cm}$ long, with $15 \mathrm{~cm}$ diameter. Presumably, the contraction of pelvic floor muscles can be promoted by sitting on this small foam roller and performing various breathing exercises while devoting attention to pelvic floor muscles. Nevertheless, its efficacy has not been verified. To verify the usefulness of the small foam roller on the contraction of pelvic floor muscles, this study was conducted to demonstrate the effects of various breathing exercises performed by a person while sitting on the roller.

\section{PARTICIPANTS AND METHODS}

Participants were 10 healthy males (age $19.9 \pm 1.6$ years, $173.3 \pm 4.8 \mathrm{~cm}, 70.6 \pm 12.3 \mathrm{~kg}$ ). This study was performed with the approval of the Research Ethics Committee of Juntendo University Faculty of Health and Sports Science (Approval No. 27-1) after providing participants proper oral and written explanations of the objective, details, and procedures of the study, in addition to possible risks and measures to be taken and compensation. Informed consent was received from each participant before measurement.

Experimental procedures in this study are presented below. Participants received $500 \mathrm{ml}$ of water after urinating first. Then they waited at rest in a sitting position until they felt an urge to urinate. Measurements were taken before the experiment when participants felt an urge to urinate. This made it possible to show the position of the bladder and to evaluate the pelvic floor muscle contraction accurately. After the end of ultrasonic measurement, they waited continuously at rest in a sitting position for $10 \mathrm{~min}$ as control (CON) participants. Subsequently measurements were taken in the same way as those before the experiment. After an interval of at least 1 week, participants received $500 \mathrm{ml}$ of water after urination. Then, after they felt an urge to urinate, ultrasonic measurements were taken as before for the CON condition. Then, as a breathing exercise (EXE) condition, they sat on a small foam roller (20.5 cm length, $4 \mathrm{~cm}$ diameter) placed on a chair (Fig. 1), and performed breathing exercises of seven kinds (Fig. 2), consisting of a combination of the movement of upper limbs, devoting particular attention to the contraction of pelvic floor muscles along with breathing. Participants inhaled through the nose and exhaled through the mouth, devoting attention to the contraction of pelvic floor muscles during exhalation. Ultrasonic measurements were taken again thereafter. Measurements were taken again only under the EXE condition after an additional 1 month.

The contraction of pelvic floor muscles was recorded on video using a diagnostic ultrasound imaging system (Noblus; Hitachi-Aloka) as described in reports of earlier studies ${ }^{9-11)}$. Imaging positions were set as a supine position with $60^{\circ}$ and $90^{\circ}$ bending, respectively, for the hip joint and the knee joint. The ultrasonic frequency was set as $3.0 \mathrm{MHz}$. The probe was placed in a position to image the bladder using the upper side of the pubic symphysis as the mark. The contraction of pelvic floor muscles was evaluated based on the movement of the posterior side of the bladder. Participants repeated the contraction and relaxation of pelvic floor muscles three times each and six times in all in each measurement under the oral direction of an experimenter. The oral instruction was unified as "please tighten the anus and pull it inside."

Three still images each, taken when pelvic floor muscles relaxed and contracted, were extracted from the recorded video. Then, using the still images to evaluate the pelvic floor muscle contraction, the movement distance (mm) of the posterior side of the bladder was calculated as the difference between the distance from the abdominal wall to the posterior side of the bladder when relaxed and that from the abdominal wall to the posterior side of the bladder when contracted (Fig. 3).

Subjective evaluation was performed using the Numerical Rating Scale (NRS) to assess whether pelvic floor muscles can be contracted before the experiment and after the end of the experiment under the CON condition and the EXE condition. The NRS self-evaluation score indicated the "no contraction at all" state as (0) and the "contracted to the maximum" state as (10) in this study. Also, how well the pelvic floor muscles contracted in the measurement was evaluated using 11 levels from 0 to 10 .

Paired t-tests were used to test the differences before and after experimentation to evaluate pelvic floor muscle contraction and NRS under the CON condition. Moreover, for the EXE condition, a generalized linear model was used to evaluate pelvic floor muscle contraction; NRS was used to assess effects after the experiment and 1 month after the experiment. Additionally, significance was inferred for probability of less than 5\%. Software (SPSS ver.24; SPSS Inc.) was used for all analyses.

\section{RESULTS}

No difference was found under the CON condition: $2.5 \pm 1.0 \mathrm{~mm}$ before the experiment and $2.9 \pm 2.3 \mathrm{~mm}$ after the experiment. Under the EXE condition, the movement distances were $5.6 \pm 2.5 \mathrm{~mm}$ before the experiment, $13.7 \pm 5.2 \mathrm{~mm}$ after the experiment, and $9.6 \pm 3.7 \mathrm{~mm}$ at 1 month after the experiment. Results show that the movement distance had increased $(p<0.001)$ immediately after EXE, and had decreased $(p<0.001)$ after 1 month, although results remained higher than before the experiment $(\mathrm{p}<0.001)$.

Subjective evaluations (NRS) were $3.1 \pm 1.1$ before the experiment and $3.3 \pm 1.3$ after the experiment under the CON 

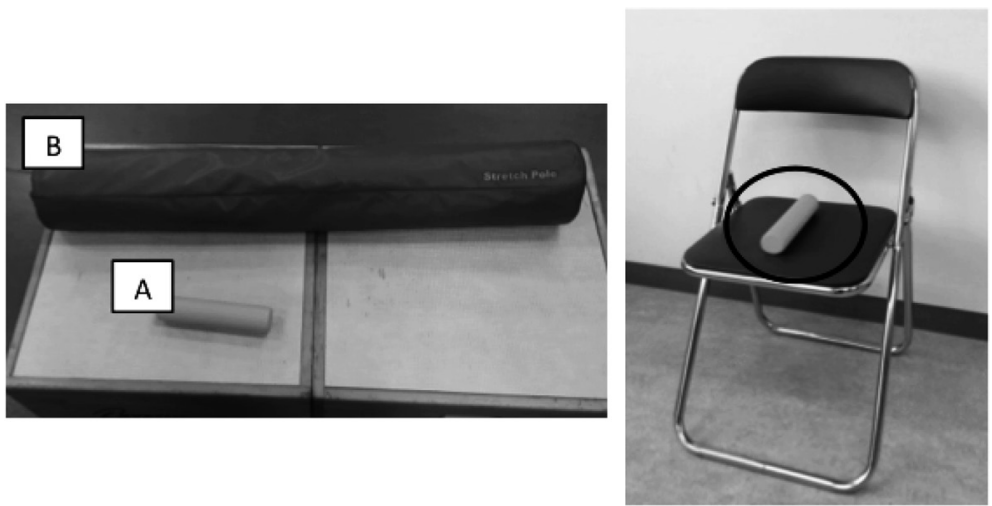

Fig. 1. A, Soft and durable cylinder as a small polyurethane foam roller (stretch pole) used for this study $(20.5 \mathrm{~cm}$ length, $4 \mathrm{~cm}$ diameter). B, Ordinary foam roller (StretchPole ${ }^{\circledR} ; \mathrm{LDN}, 98 \mathrm{~cm}$ length, $15 \mathrm{~cm}$ diameter, foamed olefin resin with a synthetic cover). As shown at right, a small stretch pole (20.5 cm length, $4 \mathrm{~cm}$ diameter) was used and placed on a chair.
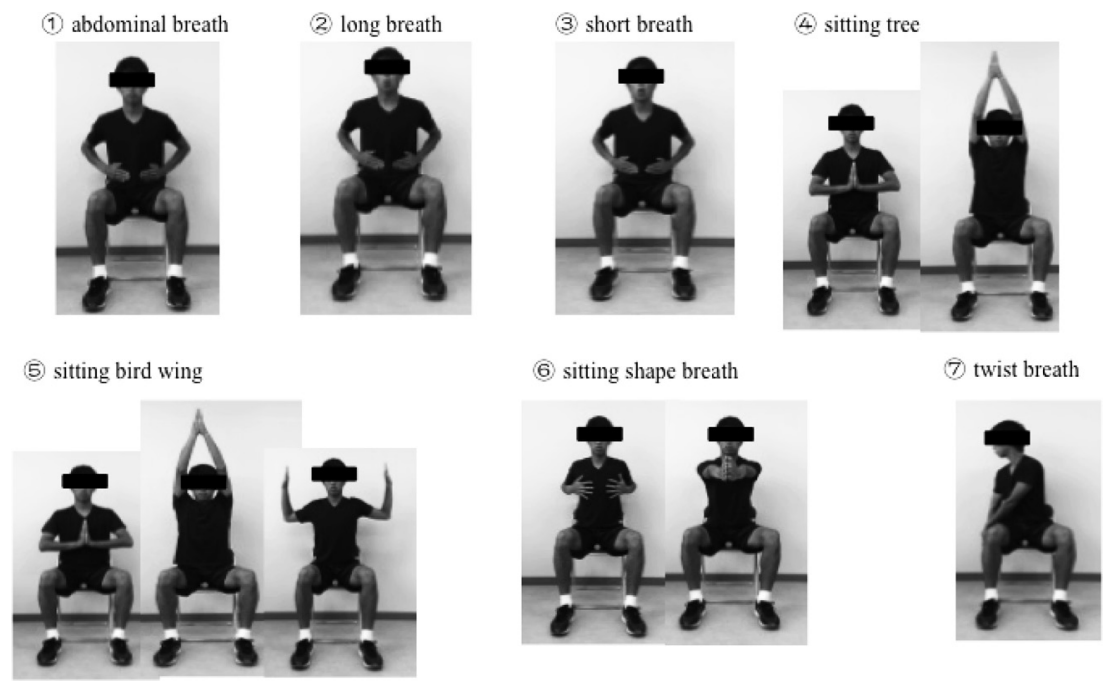

Fig. 2. Breathing exercises of seven kinds.
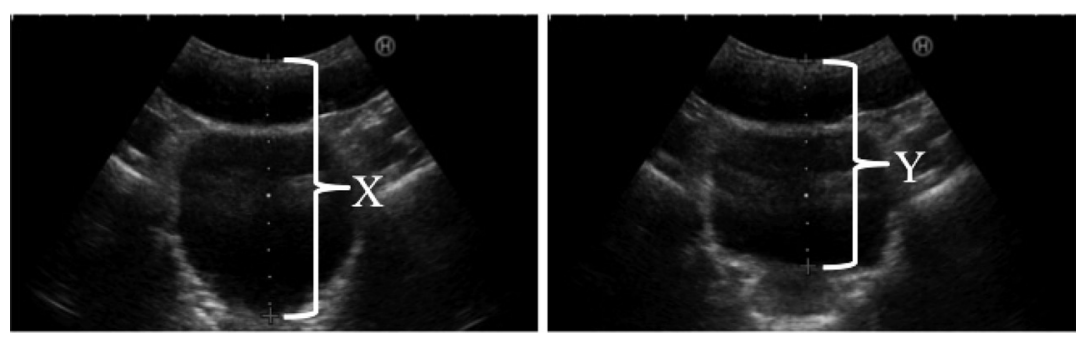

Fig. 3. Evaluation of pelvic floor muscle contraction. Distances between the back face of bladder to the stomach wall were measured when pelvic floor muscles relaxed $(\mathrm{X})$ and contracted $(\mathrm{Y})$. To evaluate the pelvic floor muscle contraction, the moving distance $(\mathrm{mm})$ of back face of bladder was measured $(=\mathrm{X}-\mathrm{Y})$.

condition. Under the EXE condition, subjective evaluations were $3.6 \pm 1.8$ before the experiment, $8.2 \pm 1.5$ after the experiment, and $6.1 \pm 1.4$ at 1 month after the experiment. Results show that a feeling of contraction was a stronger $(\mathrm{p}<0.001)$ immediately after EXE. Moreover, that feeling remained stronger after 1 month $(p<0.001)$, although it was weaker than that immediately after the experiment $(p<0.001)$. 


\section{DISCUSSION}

Results of this study demonstrated that pelvic floor muscle contraction was promoted by breathing exercises of seven kinds performed using a small foam roller, at least in males. Results of subjective evaluation also show that the feeling of contraction was stronger immediately after the end of exercise. Moreover, these effects persisted for 1 month, but the effects might be attenuated compared to those reported immediately after exercise $(5.6 \pm 2.5 \mathrm{~mm}$ before the experiment, $13.7 \pm$ $5.2 \mathrm{~mm}$ after the experiment, and $9.6 \pm 3.7 \mathrm{~mm}$ at 1 month after the experiment). Snyder and Fry ${ }^{12)}$ reported that when attention is devoted to the contraction of a specific muscle by oral direction during exercise, the activity of the directed muscle increases significantly. The present study used no oral direction to direct attention to contraction during breathing exercises, but the small stretch pole was considered to play that role. Pelvic floor muscles are regarded as being continuously in a pressed state by sitting on this stretch pole, making it possible to contract pelvic floor muscles consciously during exercise, resulting in the promotion of pelvic floor muscle contraction. It was originally planned in this study to conduct experiments with females of the same age group to compare effects for males and females. However, effects of contraction promotion were not observed in females in the experiment (reference data: $6.7 \pm 3.7 \mathrm{~mm}$ before the experiment, $7.1 \pm 3.4 \mathrm{~mm}$ after the experiment, and $5.8 \pm 3.2 \mathrm{~mm}$ at 1 month after the experiment, showing no change by EXE immediately after or 1 month after). A possible factor is that an excessive urge to urinate related to the experimental procedures in this study inhibited the pelvic floor muscle contraction. With that, the change over time in the urge to urinate and the pelvic floor muscle contraction was investigated in 1 woman participant. As a result, it was observed that the urge to urinate increased while the level of the contraction of pelvic floor muscles decreased over time. In this study, the pelvic floor muscle contraction was evaluated using the movement distance of the posterior side of the bladder. Additionally, it was necessary to store water in the bladder to check the posterior side of the bladder with a diagnostic ultrasound imaging system. It is expected that not a few female participants were measured while feeling a strong urge to urinate; it is possible that they could not contract pelvic floor muscles properly, thereby showing results of 'no effect'.

Incontinence is a health problem that is often observed in females. Reportedly, $35 \%$ of females aged 18 years or older suffer incontinence ${ }^{13}$. Moreover, the prevalence of stress urinary incontinence is higher among them. Pelvic floor muscles including the sphincter are important to prevent incontinence based on the anatomical structure ${ }^{14)}$. For that reason, the method used for this study is expected to be effective for the prevention and improvement of stress urinary incontinence. Nevertheless, because no clear effect was demonstrated for females, it is necessary to revise the measurement method and perform further verification.

It has also been reported that pelvic floor muscles, in coordination with other muscles that comprise the inner unit, contribute to trunk stability and that exercise for pelvic floor muscles is also useful for the improvement of lower back pain ${ }^{8)}$. Therefore, it is possible that the breathing exercises used for this study engender the prevention and improvement of lower back pain. Moreover, the simplicity of exercise is an important factor that must be considered for exercise to be performed continuously. Exercises for pelvic floor muscles have been done using various methods before. However, such exercises could not always be performed smoothly by everyone. By contrast, the method used for this study is extremely simple: one need only sit on the small foam roller placed on a chair while performing breathing exercises. The method can therefore be performed by everyone smoothly and safely. It is likely to be useful for middle-aged and elderly people.

In conclusion, various exercises performed while sitting on a small stretch pole promoted the voluntary contraction of pelvic floor muscles. In addition, its efficacy was demonstrated at least in young males.

Finally, results of this study were presented at The 2016 Sports Medicine Australia Conference (J. Sci. Med. Sport, 2017, 20: e44-e45).

\section{Funding and Conflict of interest}

The authors have no conflict of interest, financial or otherwise, in relation to this study.

\section{REFERENCES}

1) Vleeming A, Pool-Goudzwaard AL, Stoeckart R, et al.: The posterior layer of the thoracolumbar fascia. Its function in load transfer from spine to legs. Spine, 1995, 20: 753-758. [Medline] [CrossRef]

2) Faries MD, Greenwood M: Core training: stabilizing the confusion. Strength Condit J, 2007, 29: 10-25.

3) Kibler WB, Press J, Sciascia A: The role of core stability in athletic function. Sports Med, 2006, 36: 189-198. [Medline] [CrossRef]

4) Nesser TW, Huxel KC, Tincher JL, et al.: The relationship between core stability and performance in division I football players. J Strength Cond Res, 2008, 22: 1750-1754. [Medline] [CrossRef]

5) Willardson JM: Core stability training: applications to sports conditioning programs. J Strength Cond Res, 2007, 21: 979-985. [Medline]

6) Willson JD, Dougherty CP, Ireland ML, et al.: Core stability and its relationship to lower extremity function and injury. J Am Acad Orthop Surg, 2005, 13: 316-325. [Medline] [CrossRef]

7) Kim E, Lee H: The effects of deep abdominal muscle strengthening exercises on respiratory function and lumbar stability. J Phys Ther Sci, 2013, 25: 663-665. 
[Medline] [CrossRef]

8) Sapsford RR, Hodges PW, Richardson CA, et al.: Co-activation of the abdominal and pelvic floor muscles during voluntary exercises. Neurourol Urodyn, 2001, 20: 31-42. [Medline] [CrossRef]

9) Bø K, Sherburn M, Allen T: Transabdominal ultrasound measurement of pelvic floor muscle activity when activated directly or via a transversus abdominis muscle contraction. Neurourol Urodyn, 2003, 22: 582-588. [Medline] [CrossRef]

10) Dietz HP, Wilson PD, Clarke B: The use of perineal ultrasound to quantify levator activity and teach pelvic floor muscle exercises. Int Urogynecol J Pelvic Floor Dysfunct, 2001, 12: 166-168, discussion 168-169. [Medline] [CrossRef]

11) Thompson JA, O'Sullivan PB, Briffa NK, et al.: Assessment of voluntary pelvic floor muscle contraction in continent and incontinent women using transperineal ultrasound, manual muscle testing and vaginal squeeze pressure measurements. Int Urogynecol J Pelvic Floor Dysfunct, 2006, 17: 624-630. [Medline] [CrossRef]

12) Snyder BJ, Fry WR: Effect of verbal instruction on muscle activity during the bench press exercise. J Strength Cond Res, 2012, 26: 2394-2400. [Medline] [CrossRef]

13) Hunskaar S, Lose G, Sykes D, et al.: The prevalence of urinary incontinence in women in four European countries. BJU Int, 2004, 93: 324-330. [Medline] [CrossRef]

14) Ashton-Miller JA, DeLancey JO: Functional anatomy of the female pelvic floor. Ann N Y Acad Sci, 2007, 1101: 266-296. [Medline] [CrossRef] 